\title{
Les objets rituels des sociétés initiatiques chez les Wuli du Cameroun Occidental
}

The Ritual Objects of Wuli Initiatory Societies (Western Cameroon)

\section{Viviane Baeke}

\section{OpenEdition}

1 Journals

Édition électronique

URL : https://journals.openedition.org/span/1061

DOI : 10.4000/span.1061

ISSN : 2268-1558

Éditeur

École pratique des hautes études. Sciences humaines

\section{Édition imprimée}

Date de publication : 1 septembre 1987

Pagination : 177-200

ISSN : 0294-7080

\section{Référence électronique}

Viviane Baeke, «Les objets rituels des sociétés initiatiques chez les Wuli du Cameroun Occidental », Systèmes de pensée en Afrique noire [En ligne], 8| 1987, mis en ligne le 07 octobre 2013, consulté le 21 septembre 2021. URL : http://journals.openedition.org/span/1061 ; DOI : https://doi.org/10.4000/ span. 1061 


\title{
LES OBJETS RITUELS DES SOCIETES INITIATIQUES
}

\section{GHEZ LES WULI DU GAMEROUN OCCIDENTAL*}

\author{
par
}

\section{Viviane Baeke}

\section{INTRODUCTION}

Les Wuli, communauté patrilinéaire et patrilocale d'environ 4000 personnes, vivent aux confins septentrionaux du monde bantou, à la frontière entre le Cameroun occidental et le Nigéria. Ils sont rattachés au groupe linguistique Mbam-Nkam auquel appartiennent également les Bamoum et les Bamileke. Mais à la différence de leurs célèbres voisins, ils ont toujours ignoré l'aventure politique de la chefferie.

La pensée religieuse wuli se préoccupe surtout d'organiser en un système cohérent les origines mythiques comme les causes surnaturelles des malheurs tels que les mauvaises récoltes, la maladie ou la mort. Ils dédaignent la communication avec les ancêtres et confient les principales fonctions magico-religieuses à des sociétés initiatiques qui constitueront l'objet principal de notre propos.

Le mythe d'origine relate le début de l'aventure humaine sur terre. Au commencement des temps, deux démiurges s'opposent violemment par le feu et l'eau dans le but de conquérir la mâttrise suprême :

* Nous avons adopté, par rapport à l'alphabet phonétique utilisé par l'IAI, une graphie simplifiée : $\varepsilon=\grave{e}, \gamma=g h, \partial=\ddot{e}, \int=\mathrm{ch}$, $\eta=n g, \tilde{a}=$ an. 
créer, façonner I'univers des êtres vivants. Ces deux adversaires sont Nui Ndü, le Génie de I'Eau et Nui Mank'a, le Génie de la sorcellerie. A l'issue de ce combat dont le Mastre de l'Eau sort vainqueur, les premiers hommes surgissent du fond d'une rivière. Le mythe cesse alors de parler d'un Génie de l'Eau unique et chaque cours d'eau devient le lieu de résidence de plusieurs génies nui ndü dont les villages, semblables à ceux des hommes, sont enfouis au fond des trous d'eau. L'adversaire malchanceux du Génie de l'Eau dans le mythe d'origine reste cependant un redoutable ennemi de la société wuli : mâtre de la sorcellerie, il peut insuffler à certains êtres humains lorsqu'ils sont encore à l'état embryonnaire des pouvoirs surnaturels maléfiques. Ces différentes formes de sorcellerie sont à l'origine de beaucoup de malheurs.

On appelle Rè la sorcellerie la plus dangereuse, celle qui provoque des maladies mortelles. Le sorcier Rè se transforme la nuit en chouette, en léopard ou en chien et se rend au chevet de sa victime endormie pour lui inoculer une maladie. Le Génie de la sorcellerie est aussi à l'origine de toute une série de pouvoirs qui portent chacun le nom de l'animal utilisé comme véhicule par le sorcier : l'échassier gris (fo) vole le sorgho ou le maìs, l'aigle et le python chapardent les poules, I'hippopotame saccage les récoltes de légumes sur les berges des rivières, le serpent aquatique, friand de poissons, détruit les ponts de liane, etc. Le Génie de la sorcellerie choisit au hasard un foetus humain et le manipule de manière à créer une anomalie physiologique qui devient le siège d'une des facultés maléfiques mentionnées ci-dessus. Seule l'autopsie permet d'en révéler l'existence ${ }^{1}$. Créer ces "malformations" est la seule intervention directe du Génie maléfique dans l'univers des hommes.

${ }^{1}$ Dans le cas de la sorcellerie Rè, il s'agit de l'auricule de l'oreillette du coeur qui affecte la forme d'une crête de coq, appelée ghëlëngu. Les autres types de sorcellerie sont associés à des organes différents. Par exemple, fo est provoqué par l'existence de trois espèces de sacs mous et blancs, soit lovés dans l'utérus des femmes, soit fixés près du foie des hommes. 
En revanche, les génies de l'eau se mêlent aux activités humaines de bien des manières. Responsables de la pluie, de la fécondité des femmes et de l'abondance du gibier, les génies aquatiques sont aussi à l'origine du savoir et des techniques. Les hommes entretiennent avec eux des relations de bonne intelligence et certains racontent comment ils ont été initiés à une technique particulière lors d'une rencontre avec un génie, au détour d'un chemin forestier.

Mais si les nui ndü sont sans cesse évoqués dans la vie quotidienne des Wuli, c'est surtout parce qu'ils prêtent main forte aux humains dans leur lutte incessante contre la sorcellerie. Face au pouvoir maléfique Rè, les génies de l'eau confèrent à certains foetus un contre-pouvoir, inné et surnaturel, appelé mantacho. Ce pouvoir de nature divinatoire permet à ceux qui le possèdent de se mouvoir dans le monde nocturne des sorciers Rè et de détecter soit leur présence au sein d'une assemblée, soit leurs activités. L'hommemantacho a la faculté de se transformer en abeille, papillon ou chauve-souris afin de faciliter son enquête divinatoire. Il peut également exécuter un rituel destiné à tuer un sorcier (Baeke, 1984: 171). Néanmoins il n'exerce ses talents que lorsque la mort est sur le point de frapper ou vient de faire son oeuvre, soit pour conjurer l'inéluctable, soit pour découvrir et punir les sorciers Rè coupables de meurtre. En dehors de ces cas extrêmes, on fera appel, pour enrayer le maléfice, à d'autres anti-sorciers : les membres des sociétés initiatiques Rö qui occupent le premier rang dans les activités rituelles. Leur fonction, préventive et prophylactique, consiste à la fois à dissuader les sorciers d'intervenir et à guérir les maladies provoquées par ceux-ci. Ce pouvoir Rö est conféré, comme le pouvoir divinatoire mantacho décrit plus haut, par les génies de l'eau. Cette fois, ils interviennent par l'intermédiaire d'objets de toutes espèces appartenant aux sociétés Rö. Ces objets eux-mêmes appartiennent à la sphère du Rö. 


\section{LES "CHOSES" DU RÖ : RÖVÖ}

\section{A. Le monde surnaturel et le Rö}

Les Wuli possèdent sept sociétés initiatiques Rö. Leurs membres sont toujours des hommes. Ils tirent leur pouvoir de la possession et/ou de l'utilisation d'objets consacrés, les Rövö ("choses du Rö") qui doivent rester cachés aux yeux des non-initiés, des femmes et des enfants. Ces objets reposent dans des sanctuaires appelés Khya Rö. Au sein du groupe des initiés d'une société, une distinction est faite entre les détenteurs des objets Rö et ceux qui ne font qu'utiliser leurs pouvoirs. Lors de l'initiation à une société, on dit uniquement des premiers qu'ils "épousent" (wu ngwazë) les objets Rö, tandis que les seconds comme les premiers "payent le prix" (mi) de leur admission. Le processus d'initiation des uns et des autres est toutefois semblable.

Ces sept sociétés diffèrent par le nombre de leurs membres, I'importance des pouvoirs quion leur attribue et la nature des objets qu'elles manipulent. Elles poursuivent cependant toutes deux buts communs : protéger les villageois, individuellement ou collectivement, contre la sorcellerie et empêcher les transgressions telles que le vol ou les querelles. En outre, chacune des sept sociétés possède des charmes spécifiques et peut envoyer aux sorciers et aux transgresseurs une ou plusieurs maladies particulières, selon un code nosologique précis.

J'ai décrit ailleurs en détail les mécanismes par lesquels les membres des sociétés initiatiques acquièrent leur pouvoir (Baeke, 1984: 171). Reprenons-en les traits les plus pertinents. Au cours de l'initiation, les nouveaux membres acquièrent leur pouvoir magique par un sacrifice qui les associe étroitement aux objets du Rö, les Rövö. Ce pouvoir leur permet dès lors d'utiliser des "charmes" - distincts des Rövö, objets cultuellement élaborés - qui sont en général des plantes de la brousse ; investies indirectement du pouvoir Rö, elles serviront à protéger le village, à guérir une maladie ou à la provoquer. Cette dernière intervention est radicalement distincte de l'action Rè dans la mesure où elle ne peut 
viser qu'un sorcier ou un transgresseur. Contrairement à l'action illégitime et aveugle de la sorcellerie Rè, la contre-sorcellerie associée aux plantes du Rö s'exerce toujours selon des critères de légitimité.

Revenons-en aux "choses du Rö", objet central de notre propos. S'agit-il de fétiches ? Examinons leur statut.

Ces objets, en nombre fini pour chaque société, sont en principe éternels. Mais ils doivent être remplacés lorsqu'ils se cassent ou perdent accidentellement leur pouvoir. Il s'agit alors d'activer le nouvel objet en mettant en branle un mécanisme complexe. Les Wuli sont formels sur ce point : qu'il s'agisse d'une cloche de fer, d'une statuette de terre cuite ou d'une calebasse-instrument musical, l'objet en tant que tel ne possède aucun pouvoir ; il n'est encore qu'une enveloppe vide, sans "esprit", inopérant. Le rite d'investiture de l'objet consiste notamment en un sacrifice de poulet qui le transforme en Rövö, "chose du Rö", c'est-à-dire un lieu où cohabitent différentes instances surnaturelles : un esprit de l'eau bénéfique et une "lignée" de fantômes d'origine maléfique, les mank'a. Essayons d'y voir clair.

Nous avons vu que les génies de 1 'eau sont des êtres surnaturels, à l'image de l'homme, qui vivent en villages au fond des rivières. La forêt et les cours d'eau sont leurs domaines privilégiés d'où ils observent le monde des hommes qui entretiennent avec eux des relations de bonne intelligence. Ces génies de l'eau sont présents au village dans les objets Rö au sein desquels ils ont été invités à entrer lors d'un rituel (rituel de naissance ou d'activation de l'objet Rö). Ils ne portent pas des noms spécifiques mais, pour chaque société Rö, les génies qui lui sont associés proviennent d'une rivière particulière. Souvent lointaine et au nom oublié, celle-ci est censée couler dans le territoire d'origine de la société elle-même.

La deuxième composante spirituelle des objets Rö est une lignée đe mank'a (fantômes) qui relève paradoxalement du domaine de la sorcellerie. Nui Mank'a, génie de la sorcellerie et rival du génie de l'eau primordial Nui Ndï, est à l'origine du processus compliqué de la création des fantômes mank'a. Voyons-en les étapes. 
Nui Mank'a attribue à certains humains pris au hasard et qui resteront anonymes le pouvoir de sorcellerie Rè. Ces sorciers, à leur tour, secrètent à la mort de tout être humain, homme, femme ou enfant, une sorte d'enveloppe blanche, réplique fantôme du défunt, invisible aux non-sorciers, appelée mank'a. Ce fantôme hante les lieux des funérailles et effraie les vivants. Il disparât généralement au bout de quelques jours et va rejoindre en brousse le zi ou principe vital du défunt au sein duquel il se fond alors. Ce principe éphémère, ultime malédiction que les sorciers projettent dans le monde des vivants lorsqu'un homme ou une femme bascule dans la mort, ne possède pourtant aucun pouvoir maléfique ou bénéfique. "Ils ne font qu'effrayer", disent les Wuli. Pour comprendre la nature de cette frayeur, il faut se tourner vers les mécanismes d'ensorcellement. La sorcellerie Rè ne s'exerce que sur des personnes prises à partie en public par le discours d'un tiers. Menace, serment de vengeance, médisance ou calomnie, ces propos captés par l'oreilie d'un sorcier sont I'unique brèche au travers de laquelle peut s'exercer son action destructrice. A la mort de l'auteur de ces paroles, les sorciers secrèteront son mank'a et le feront errer sur l'aire funéraire. Ce fantôme, quoique invisible, est perçu grâce à l'odeur d'huile de palme qu'il dégage ou le courant d'air qu'il provoque en se déplaçant. Il effraie les vivants parce qu'il est le symbole du pouvoir des sorciers de se souvenir des paroles qu'a prononcées le mort. Son fantôme planant lors des funérailies rappelle aux vivants que toutes les accusations, menaces ou malédictions proférées par le défunt restent inscrites dans la mémoire des sorciers et continueront à conduire leurs actes maléfiques futurs.

Mais si un initié détenteur a'objets Rö meurt, cette entité post-mortem qu'est son mank'a sera captée, emprisonnée par les génies de l'eau à l'intérieur des Rövö du défunt où elle ira rejoindre les mank'a des ascendants du mort, c'est-à-dire les fantômes de son père, grand-père, etc., qui ont été en possession de ces mêmes objets avant lui. Ceci explique que les mank'a littéralement incorporés au Rö constituent une Iignée patrilinéaire. Normalement, un mank'a erre en brousse en compagnie du zi, l'autre principe spirituel qui s'échappe du corps d'un mort; il est inoffensif et 
ne possède aucun pouvoir. Mais lorsque l'un d'eux est incorporé à un objet Rö, il change de nature et devient apte à s'attaquer aux sorciers et aux transgresseurs. Capté et domestiqué par un esprit de l'eau, ce fantôme se retourne contre la source même de sa création, la sorcellerie. Mais son pouvoir, au contraire de celui, illicite et incontrôlable, des sorciers, sera assujetti aux lois qui régissent les sociétés initiatiques et dirigé uniquement contre ceux qui transgressent ces règles socialement approuvées.

Les "choses du Rö" doivent donc leur efficacité à deux principes complémentaires - fantômes et esprit de l'eau - qui, en dehors des activités rituelles des sociétés initiatiques, sont foncièrement antinomiques. Le mythe de création oppose en effet (cf. plus haut) le premier génie de l'eau et le créateur de la sorcellerie qui est à l'origine de l'existence des fantômes maléfiques. Ces fantômes deviennent les alliés des esprits de l'eau : ils passent de la sphère du Rè à la sphère du Rö.

L'ambivalence du pouvoir Rö se déchiffre aussi dans l'action spécifique qu'exerce chacune des deux entités constitutives de cette puissance. Une "chose du Rö" a aussi bien le pouvoir d'infliger une maladie que de provoquer sa guérison, mais en fait une division du travail s'instaure : les fantômes envoient les maladies, le génie de l'eau guérit. Vis-à-vis des profanes, les initiés mettent rarement en relief cette dualité ; les différentes formes du pouvoir émanant des objets se conçoivent globalement, comme un tout cohérent, le Rö. Lorsqu'un patient entre en rapport avec un initié, la puissance Rö que celui-ci manipule est considérée comme indivisible. En revanche, sa dualité apparât dans les rites qui ne mettent en jeu que des initiés et notamment dans le rite d'activation. A cette occasion, les génies de l'eau et les fantômes mank'a s'incorporent à un nouvel objet façonné pour en remplacer un ancien devenu inefficace. Ce rite comporte deux étapes consécutives importantes. Le "Père du Rö" répand le sang du bec d'un poulet sur le futur réceptacle et $y$ fixe une boulette de résine ya ${ }^{2}$ qu'il enflamme immédiatement provoquant un dégagement de fumée noire.

2Résine provenant de l'arbre të yar : Burseraceae Dacryodes. 
Ces deux étapes rituelles distinctes concernent, soulignons-le encore une fois, deux entités spirituelles différentes : le sang invite l'esprit de l'eau à entrer dans l'objet, la fumée dégagée par la résine est destinée au contraire à retarder l'incorporation des fantômes au fétiche. Il faut, disent les Wuli, que le génie de l'eau entre le premier dans le Rövö, que les mank'a le rejoignent plus tard ${ }^{3}$. On comprend mieux ainsi que l'écoulement du sang et le dégagement de fumée soient quasiment simultanés.

Tant que dure le rite d'activation de l'objet, seul un génie de l'eau l'anime, les fantômes étant écartés, tenus à distance par la fumée noire qui les effraie. Pendant que la résine brûle, l'officiant prononce la formule suivante : "Nui Naü, agis ! Nui Mank'a, ne t'en occupe pas !". La raison pour laquelle les mank'a doivent être temporairement écartés de leur nouveau support lors de la "naissance" d'un fétiche ne m'a pas été donnée, mais un autre rituel nous aide à mieux comprendre la situation. Il s'agit d'un rituel de protection collectif qui fait également intervenir la résine enflammée pour éloigner temporairement les fantômes mank'a de leur support Rövö. Ce rite (kö manzë, fermer, barrer la route) est effectué lorsqu'une maladie grave d'origine maléfique frappe de manière récurrente et menace de se transformer en épidémie. Les villageois sollicitent l'aide des membres d'une société Rö particulière, la société Wankya (réputée la plus puissante), afin de barrer aux sorciers les routes menant de la brousse vers le village ou inversement. Pour cela, les principaux initiés de Wankya vont transférer leurs objets consacrés, une série de figurines de terre cuite, aux points d'entrée du village et aux croisées des chemins marqués les uns et les autres par des pierres dressées. On y place une série de charmes végétaux que la présence même des Rövö vivifie. Les initiés rameneront ensuite ceux-ci dans leur sanctuaire, tandis que ceux-là resteront sur place. Tout sorcier animé d'intentions

${ }^{3}$ La fumée dégagée par la combustion de la résine est censée effrayer et éloigner les fantômes mank'a aussi bien lors de ce rite d'activation d'un fétiche que dans d'autres contextes, par exemple lorsque le mank'a errant du défunt hante les lieux de funérailles. 
maléfiques et traversant cette barrière magique sera tôt ou tard atteint par la maladie de Wankya (ventre gonflé, ascite) et en mourra.

La veille de ce rite, le détenteur des figurines les sort du sanctuaire et les dispose au pied d'une pierre plate (mwu de Wankya), symbole puissant de la société Wankya puisqu'elle aussi est habitée par les génies de l'eau et les fantômes et qu'elle intervient dans les rites d'initiation et certains rites de guérison. Le "Père de Wankya" passera la nuit sur le lieu même. A l'aube, entouré des autres initiés, il enflammera une boulette de résine yan posée sur la pierre consacrée à Wankya ; tous tendent leurs mains au-dessus de la fumée ainsi produite et se frottent le visage. Le détenteur des figurines Rö éteint ensuite le feu en l'arrosant de vin de palme.

Les Wuli commentent de la manière suivante ce rituel préliminaire : il ne faut pas que les mank'a soient dans les Rövö pendant que les initiés les transportent jusqu'aux frontières du village ("ils suivent derrière"). Si les mank'a restaient au sein des figurines au cours de ce transfert, la santé des initiés qui les manipulent pourrait en être affectée (faiblesse générale et douleurs dans tout le corps). Lorsque les figurines réintègrent le village, les mank'a viennent s'y loger à nouveau; ils s'attaqueront tout spécialement aux sorciers auxquels les charmes qui viennent d'être déposés aux portes du village et aux croisées des chemins barrent la route.

Normalement, les initiés manipulent sans danger les Rövö au village. Mais le rituel précédent nous enseigne clairement qu'il n'en est pas de même lorsqu'on les emmène en brousse. Il faut alors désamorcer la puissance redoutable que constituent les mank'a qui risquent de se retourner contre les initiés eux-mêmes. C'est ce que confirment explicitement les commentaires de mon informateur.

L'association génie aquatique-fantômes maléfiques au sein du Rö représente donc dans certaines circonstances précises un danger incontrôlable échappant aux règles selon lesquelles le Rö fonctionne et agit habituellement. Ce danger est tel qu'il peut même affecter les initiés. Lorsqu'il se présente, il faut réduire les mank'a à l'impuissance en les expulsant des réceptacles Rö. Car un mank'a hors du Rö, donc privé du pouvoir que l'esprit de l'eau lui a octroyé, 
redevient inoffensif comme les fantômes errant lors des funérailles. Quelle est donc la nature de ce danger ?

On peut constater que les deux rituels où il est obligatoire de séparer du Rö les fantômes mank'a ont une caractéristique commune: la transition, le passage d'un état à un autre. Dans un cas, il s'agit de l'investiture d'un objet rituel nouveau, dans l'autre, du transport physique d'un objet en un autre lieu, du village vers la brousse. Il faut se souvenir ici que les mank'a sont créés par les sorciers uniquement lors du passage des hommes de la vie à la mort, moment de transition par excellence.

On peut penser que lors des situations critiques créées par les rituels précédents, l'emprise que les génies de l'eau exercent sur les fantômes mank'a se relâche et que l'équilibre génie-fantômes se rompt dans la sphère du Rö. Le pouvoir qui résulte de ce déséquilibre devient alors incontrôlable et dangereux, à l'image même des pouvoirs maléfiques des sorciers, créateurs des mank'a. La seule solution est alors d'expulser momentanément les fantômes de leurs réceptacles; écartés de leur source d'énergie que sont les esprits de l'eau au sein des objets Rö, ils redeviennent des mank'a errants et inoffensifs. Lorsque le rite marqué par la transition est terminé, les fantômes réintègrent leur support pour y jouer leur rôle en association avec les esprits de l'eau.

Que le pouvoir Rö s'altère lors des situations de transition se trouve corroboré par le fait suivant. lorsqu'un malade bascule dans l'ultime phase qui précède le trépas, c'est-à-dire le délire ou le coma, les sociétés Rö deviennent impuissantes à le protéger contre les sorciers qui l'entrainent vers la mort et s'apprêtent à sécréter son mank'a. Il faut alors faire appel à d'autres catégories d'anti-sorciers qui détiennent leurs pouvoirs uniquement des génies de l'eau, sans l'intermédiaire du Rö où ceux-ci sont associés intimement aux fantômes mank'a, ce produit ambigu de la sorcellerie. Lors de ces situations de transition, le pouvoir Rö perd de sa cohésion, échappe partiellement au contrôle des initiés et il faut alors des précautions rituelles supplémentaires pour que les hommes assurent à nouveau leur mainmise sur le surnaturel. Mais ils renouvelleront sans cesse la synthèse dangereuse de ces deux concepts 
antinomiques qui sont les génies aquatiques et les fantômes mank'a. Cette association est le prix à payer pour assurer l'efficacité du système Rö.

\section{B. Description et inventaire des nchoses du Rön}

Les pages qui précèdent montrent une relative unité dans le traitement rituel des objets à l'origine du pouvoir des sept sociétés initiatiques wuli, unité que reflètent aussi le terme générique Rö qui les englobe toutes ainsi que les activités communes de ces associations (défense contre la sorcellerie et dissuasion des transgressions). Elles se démarquent cependant les unes des autres par rapport à d'autres activités. Certaines sociétés participent à des rites saisonniers assurant la fertilité des champs. D'autres ont un rôle important à jouer lors des rites funéraires. Une autre encore est associée à la chasse.

De même, sur le plan des formes esthétiques et des matériaux utilisés, l'ensemble des Rövö se révèle plutôt hétéroclite. Les récits, mythes et anecdotes décrivant l'origine de chaque société ou les avatars ayant amené leur adoption par les Wuli se réfèrent à un amas d'événements hétérogènes, sans unité apparente. Toutefois, certains événements, puisés dans l'un ou l'autre de ces récits, permettent de construire une chronologie de l'irruption des sociétés Rö dans l'univers wuli, mince f'ragment d'histoire retravaillé par la pensée mythique qui seule en détient la mémoire. Comme ces récits sont aussi les seuls parfois à légitimer et expliquer la nature des supports matériels utilisés dans la confection des Rövö, il n'est pas inintéressant d'en donner ici un bref aperçu.

1. Naissance des sociétés Nkè et Tsimbi

Ce sont les sociétés Nkè et Tsimbi qui furent les premières à être créées et voici pourquoi. Tout au début des temps, Nui Ndü fit sortir d'un trou d'eau les deux premiers hommes, Pwopwo et Sasa, puis leur procura à chacun une épouse. Mais l'épouse de Sasa fut paresseuse et capricieuse, refusant de faire la cuisine et 
passant la journée juchée sur le dos de son mari. Aussi les deux frères l'enivrèrent-ils, Pwopwo l'arracha du dos de son mari et ils la déposèrent endormie dans la maison à laquelle ils mirent le feu. L'épouse de Sasa brûla avec la maison et sur ses cendres les deux frères plantèrent des graines de calebassier.

Le temps passa et les hommes se multiplièrent. Lorsque les plants de calebasse eurent grandi, ils en utilisèrent les fruits à toutes sortes d'usages : calebasse à vin, à huile, pour contenir la nourriture ou encore pour la préparer, tels ces fragments rectangulaires, appelés wibè, qui servent de louche. Ce que les villageois ignoraient c'est que tous les matins lorsqu'ils quittaient le village pour les champs, ces différentes calebasses se transformaient en êtres humains et, sous la direction des wibè jouant du tambour, tous dansaient et chantaient. Mais un enfant découvrit le secret des calebasses et les hommes, se rappelant l'histoire de l'épouse de Sasa, les rassemblèrent et y mirent le feu. Toutes les calebasses furent brûlées à l'exception d'un couple de wibè qui, s'échappant en brousse, se réfugia dans une termitière. Celles-là ne tardèrent pas, par ruse, à provoquer la guerre entre les Wuli et les habitants du village proche de Kom4. Les wibè se nourrissaient des cadavres laissés sur le champ de bataille. Ici encore la sagesse des hommes finit par déjouer leurs plans. Les deux wibè furent détruits définitivement et la paix se rétablit entre les deux villages. Après cet épisode hostile, les hommes fondèrent les sociétés Isimbi et Nkè dont les Rövö sont forgés respectivement à partir de deux espèces de calebassiers : mbë et ngë5

Aujourd'hui leur sont associées intimement les deux sociétés Chu et Bankha dont les Rövö sont respectivement un masque de fibres tressées, noir et blanc, masculin, et un masque de fibres, noir, féminin. Mais les Wuli assurent que ces deux sociétés à masques sont apparues les dernières parmi les sept sociétés Rö qu'ils possèdent.

4Kom est un village voisin, situé à trois heures de marche au sud des Wuli.

${ }^{5}$ Mbë (Tsimbi) : Lagenaria ciceroria molina ; ngë (Nkè) : non identifié, peut-être une variété de l'espèce précédente. 
Néanmoins, nous verrons au fil de la chronique wuli se dessiner une opposition entre les sociétés associées aux calebasses et aux fibres végétales d'une part et les sociétés associées au fer et à la terre cuite d'autre part. Les premières utilisent des matériaux "crus"; nous les verrons chanter et danser aux funérailles et présider un repas rituel composé de nourriture froide et crue. Les secondes, en revanche, possèdent des Rövö issus d'une cuisson par la forge ou le four. Significativement, ces sociétés, qui participent du "cuit", ne jouent aucun rôle dans le rite "cru" évoqué à l'instant. Nous allons développer cette opposition au cours de cet inventaire.

2. Apparition de la société Ntoro

Le temps passe et surgit aux portes du village un lignage dont I'aîné, Nzöro, est un forgeron renommé qui apporte aux Wuli la technique de la fonte du fer, inconnue jusqu'alors, ainsi qu'une nouvelle société Rö : Ntoro.

La société Ntoro est à bien des égards une énigme. C'est la seule association pour laquelle les Wuli peuvent préciser le lieu d'origine des esprits de l'eau qui habitent ses Rövö : il s'agit d'une rivière située sur le territoire qu'occupait précisement le lignage du forgeron Nzöro avant d'émigrer en pays wuli. Les descendants de Nzöro forment aujourd'hui le lignage Bëmëla, "gens de la forge". Actuellement, la société Ntoro existe dans beaucoup d'autres lignages, mais l'ultime étape de l'initiation d'un homme détenteur des fétiches de Ntoro se passe encore sur les berges de cette rivière, à plusieurs heures de marche au nord-ouest du village.

Les objets Rö de cette société fondée sous le signe de la forge sont d'une part un jeu de cloches liées par une corde 6 et d'autre part une cloche unique plus grande. Deux masques de bois, représentant respectivement un buffle mâle et un buffle femelle, accompagnent ces instruments de fer, ainsi que deux calebasses instruments de musique. Ces deux derniers objets Rö appartiennent

\footnotetext{
6Un processus de scission a amené aujourd'hui ce jeu de cloches à devenir les Rövo d'une société distincte appelée Mankombwu. Précisons qu'il s'agit de cloches en fer à battant externe appelées également gongs.
} 
à la fois à la société Ntoro et à deux autres sociétés. Nous reviendrons sur cette caractéristiques de sociétés initiatiques qui partagent ou échangent leurs objets Rö après avoir relaté I'histoire de la société Wankya qui est aujourd'hui l'association intiatique la plus crainte et la plus respectée des Wuli.

3. Fondation de la société Wankya

Le mythe d'origine de cette société met en scène un couple de jumeaux mixtes siamois reliés par les hanches et ne possédant chacun qu'une seule jambe. Ce couple est appelé manki mèsë ("une jambe"). Venant de la plaine de Mbaw, territoire situé à environ $50 \mathrm{~km}$ au sud-ouest du pays wuli, ils apparaissent inopinément aux portes du village. En se cachant soigneusement des femmes, ils s'installent pendant deux jours dans une concession lignagère, puis deux jours encore dans une autre. A chaque fois, leur premier acte est d'excréter dans le sanctuaire réservé aux sociétés Nkè et Tsimbi. Enfin, ils arrivent dans le village des forgerons et constatent que les sociétés Nkè et Tsimbi n'y existent pas mais que, par contre, un sanctuaire de Ntoro y est érigé. Ils s'y installent provisoirement, mais persuadent l'aîné Nzöro de construire une case rituelle qui leur soit propre. Ils séjournent chez le forgeron pendant plusieurs jours, ne manquant pas d'excréter dans le sanctuaire nouvellement construit. Le septième jour, ils rassemblent tous les hommes du lignage et annoncent leur départ. Les jumeaux confient une série de figurines de terre cuite, une double cloche et leurs excréments blancs, appelés nè, à Nzöro, l'aîné désigné comme détenteur de ces Rövö. La société Wankya est née.

Les jumeaux indiquent aussi comment trouver un substitut à leurs excréments nè lorsque ces derniers ont disparu?. Le nè est considéré comme le charme anti-sorciers le plus puissant de tous, tandis que figurines de terre et cloches métalliques sont intégrées à la catégorie des Rövö. En outre, aujourd'hui, deux statuettes de

${ }^{7}$ Concrescence mycologique blanche autour des souches pourrissantes de certains arbres : mycelium du Lentinus tuber-regium. 
bois, qui ne sortent jamais du sanctuaire, passent pour la représentation des jumeaux mixtes et, à ce titre, figurent aussi parmi les Rövö de Wankya.

La description que donnent les Wuli de ce couple "qui a deux têtes et une jambe" ressemble à celle qui font les Minyanka du Mali de certains ancêtres sefè, "premiers jumeaux de Tyèlèrè (...) soudés un à un à un tronc unique" (Jespers, 1976: p.114). Mais alors que les ancêtres jumeaux sefè sont profondément enracinés à la fois dans le passé mythique et dans le présent surnaturel des Minyanka, les jumeaux fondateurs de Wankya sont des visiteurs étrangers éphémères dont l'incursion dans l'univers wuli n'a apparemment qu'un but : imposer la société initiatique Wankya, venue d'ailleurs, par opposition à d'autres sociétés que les Wuli considèrent comme autochtones, telles Nkè et Tsimbi.

Des objets de terre cuite liés à une société initiatique ne sont, à ma connaissance, mentionnés nulle part ailleurs dans les Grassfields. Leur facture rappelle les poteries sao et surtout certaines figurines provenant des villages voisins des Wuli et de la région Mambila. L'utilisation rituelle exacte de ces figurines est rarement précisée (Gebauer, 1979: 219-223). Par contre, la double cloche, largement répandue dans toute la région des Grassfields, est un emblème toujours lié à certaines associations religieuses ou politiques (Dil1on, 1973: 305). En outre, on la retrouve, double ou simple, à travers une large part de l'Afrique, associée à la royauté, l'autorité politique ou la guerre (Vansina, 1969: 190). Ia forme élancée de cette cloche de Wankya, qu'elle soit simple ou double 8 , diffère totalement des cloches plus rectangulaires de la société Ntoro. Chez les Wuli, cette cloche possède un statut unique parmi les objets Rö : emblème public plutôt qu'objet sacré, elle est le seul objet façonné pouvant être vu et même touché par tous.

Le mythe relatant la naissance de la société Wankya met en scène un couple d'"étranges étrangers" qui, dédaignant de s'adresser aux lignages membres des sociétés de calebasses (Nkè et Tsimbi),

\footnotetext{
${ }^{8}$ Les deux modèles, simple et double, existent chez les Wuli et sont indifféremment utilisés.
} 
scellent une alliance avec les "gens de la forge" auxquels ils confient des objets de terre cuite, Rövö de la nouvelle société qu'ils introduisent chez leurs hôtes. Sur le plan mythịque, une opposition se dessine donc entre les "gens de la calebasse" et les "gens du fer et de la terre cuite".

Plus tard, selon la chronique wuli, deux nouvelles sociétés font leur apparition : Bankha et Chu dont les Rövö sont respectivement un masque de fibres féminin d'origine inconnue et un masque de fibres masculin d'origine yamba 9 . Ces deux sociétés sont devenues aujourd'hui les partenaires inséparables des sociétés à calebasses.

Une caractéristique commune lie les deux sociétés de l'origine des temps (Nkè et Tsimbi) aux deux sociétés de masques de raphia : les calebasses et les tissus de fibres sont des matériaux naturels, non traités par le feu, à l'inverse des Rövö principaux des deux sociétés Ntoro et Wankya, façonnés en fer et en terre cuite. Sur le plan de leur fabrication au moins, calebasses et fibres végétales des quatre sociétés sont du côté du "cru" par opposition aux deux autres sociétés dont les Rövö sont "cuits" par le four ou la forge.

A propos de ces deux formes de cuisson, on peut remarquer qu'elles interviennent à des étapes différentes de l'élaboration de l'objet Rö. Le minerai de fer subit la fonte avant que I'objet ne soit façonné. A l'inverse, la terre à poterie est d'abord modelée avant d'être cuite.

Il reste que ces objets associés aux jumeaux et aux forgerons ont subi une élaboration placée sous le signe du feu culturel. Qu'en est-il des calebasses et des masques de fibres ? Les matériaux qui composent les masques sont des lanières végétales ramollies par l'eau pour être ensuite tressées et tissées. Les calebasses sont évidées, rincées, trouées à plusieurs endroits pour en faire des instruments de musique. Avant toute utilisation rituelle à caractère musical, les initiés leur entourent le "cou" d'un collier tressé d'herbe nzi ${ }^{10}$. Cette herbe est le pendant terrestre d'une

9Les Wuli ne fabriquent pas le masque Chu, ils doivent l'"acheter" au village yamba de Byi. Les Yamba sont une ethnie voisine à l'est des Wuli.

${ }^{10}$ Gramineae sporbolus pyramidalis. 
plante aquatique du même nom (non identifiée) qui symbolise dans le mythe d'origine la frontière, la porte entre le monde des génies de l'eau et le monde des hommes. Lors d'un rituel de fertilité dont nous parlerons plus loin, les calebasses ainsi parées d'une plante aquatique craignent le soleil. Le rite a lieu la nuit et les calebasses regagnent leur sanctuaire avant le lever de l'astre diurne. Ainsi rituellement associées à l'eau, les calebasses se voient conférer un statut similaire à celui des fibres plongées dans l'eau même durant la fabrication des masques.

Nous pouvons donc conclure que calebasses et fibres végétales relèvent de la même catégorie symbolique en tant que produits naturels élaborés sous le signe de l'eau et non soumis à l'action du feu. Le mythe d'origine montre clairement que le feu est l.'élément destructeur des calebasses (cf. plus haut p. ). Celles-ci ne naissent pas de ce feu, elles s'y anéantissent. Ainsi s'éclaire le partage évoqué dans les mythes des objets rituels détenus par des sociétés Rö différentes.

Cette opposition entre objets placés sous le signe de l'eau et objets placés sous le signe du feu pose néanmoins un problème. Les deux sociétés détenant objets en fer et poteries possèdent aussi des objets en bois : deux statuettes représentant les jumeaux mythiques manki mesë figurent dans le matériel rituel de la société Wankya tandis que Ntoro détient un couple de masques-buffles. Qu'est-ce qui peut séparer symboliquement le bois sculpté des fibres tressées, I'une et I'autre supports naturels plus ou moins élaborés culturellement ? Probablement le processus de leur fabrication : alors que les masques de fibres peuvent être façonnés à mains nues, les objets de bois sont sculptés à l'aide d'outils de fer, produits de la forge. En outre, on dit des masques de bois qu'ils ne peuvent sortir sous la pluie. Il est donc naturel de les retrouver aux côtés du fer et de la poterie dans deux sociétés Rö. Soulignons qu'aucune autre ne possède des objets rituels en bois.

L'opposition du cru et du cuit se retrouve au plan culinaire, lors du rituel de fertilité mentionné ci-dessus et destiné à assurer la croissance du sorgho et l'abondance d'huile et de vin de palme. Ce rituel a lieu en pleine saison des pluies lorsque le sorgho 
vient d'être semé. Les sociétés Ntoro et Wankya n'y participent jamais. Les Rövö des quatre sociétés Nkè, Tsimbi, Chu et Bankha sont les acteurs principaux de ce rite au cours duquel les calebasses chantent et les masques de fibres dansent. Les chants consistent en une litanie d'exhortations enjoignant au sorgho et à toutes les plantes cultivées de croître et demandant aux insectes volants de multiplier le vin au coeur des palmiers. Un repas est préparé par les hommes ce jour-là, constitué de poisson séché, de feuilles de haricot et d'huile de palme, le tout est mangé froid et cru. Si l'on cuisait ces aliments, le jus chaud de cuisson "tuerait" le sorgho en croissance dans les champs.

Des objets Rö d'origine végétale non traités par le feu se trouvent donc symboliquement associés à une nourriture mangée froide et crue. L'emploi du feu culinaire est interdit afin de ne pas entraver la croissance des plantes cultivées.

La société Ntoro, exclue de ce rituel, est associée au monde animal et plus précisément à la chasse, par le biais d'un rituel effectué en vue de l'ouverture de la chasse, au début de la saison sèche. Les Rövö de Ntoro, deux masques de bois représentant un couple de buffles accompagnés de la cloche de fer mbanka, viennent danser toute une nuit sur l'emplacement dit "aire des têtes" où étaient conservés jadis les crânes des ennemis tués à la guerre et des léopards tués à la chasse. Les masques-buffles ne peuvent en aucun cas être touchés par la pluie. Le nom même de ces masques (ntonka) évoque la chasse à la lance (nka), pratiquée uniquement en saison sèche. Le nom du rituel auquel ils participent, ghunghë, signifie "fête de la nghë", c'est-à-dire fête de la case où sont entreposés les crânes. Si les Rövö de la société Wankya ne participent pas à ce rituel, il faut cependant souligner que certaines des figurines de terre cuite représentent ou symbolisent des animaux : le caméléon, le singe, le léopard.

Un premier groupe de quatre sociétés Rö se trouve donc associé au cru, à la saison des pluies et à la fertilité du monde végétal, tandis qu'un autre groupe de sociétés détient des objets "cuits" au four ou à la forge, ces derniers étant associés à la saison sèche et à la chasse. 
Nous avons peu parlé de la septième scciété, Mankombwu, dont les Rövö sont une série de cloches de fer liées entre elles. Rappelons que cette société est née a'tune scission : ces cloches de fer appartenaient jadis à la société Ntoro fondée par le premier forgeron. Mankombwu se trouve associée tantôt à certains rituels effectués par Ntoro dans le cadre de la lutte anti-sorcellerie, tantôt aux sociétés à calebasses et aux masques de fibres, par exemple lors des danses de funérailles. Les initiés des sociétés Chu et Bankha revêtus de leurs masques, ainsi que les membres de Nkè et Tsimbi accompagnés de leurs calebasses-instruments de musique viennent danser et chanter aux funérailles des hommes. Si le défunt est membre de Mankombur, les cloches de cette société les accompagnent. Aujourd'hui, les Wuli disent qu'elle sert surtout à entretenir l'amitié et la paix entre "beaux-frères".

Or un épisode du mythe relatant l'arrivée du lignage des premiers forgerons raconte les difficultés qu'ont eu ces derniers à s'établir au sein du village. Les hommes des lignages autochtones refusaient d'aider les forgerons à transporter leur enclume au coeur du village, obligeant ceux-ci à s'établir à ses portes. Mais bientôt des mariages eurent lieu entre filles de forgerons et hommes autochtones. Ces derniers refusaient toujours de transporter l'enclume et leurs frères essayèrent alors d'ensorceler ces femmes venant de l'extérieur, isolées de leur lignage. La riposte fut que ces épouses, filles de forgerons, transportèrent elles-mêmes l'enclume au centre du village, rétablissant ainsi la paix entre leurs pères et frères et leurs maris.

Cet épisode mythique est en fait si important que les événements qu'il raconte sont à l'origine même du nom du village et de ses habitants : Wuli signifie en effet "elles ont porté" et se réfère explicitement à l'exploit collectif des filles de forgerons qui ont porté l'enclume au sein du village.

Cette problématique de l'alliance exprimée dans le mythe, jointe à l'affirmation selon laquelle Mankombwu sert à maintenir la paix entre beaux-frères, nous conduit à penser que le processus de scission aboutissant à la séparation des cloches de Mankombwu du reste des Rövö de Ntoro pourrait être le résultat d'échanges initiatiques. Des forgerons font don à leurs gendres ou aux fils 
de ceux-ci, leurs neveux utérins, d'une partie de leur pouvoir ntoro, tandis que les sociétés Rö autochtones leur cèdent deux calebasses Rö qui désormais feront partie à la fois des sociétés de calebasses et de la société Ntoro.

Ce processus d'échange qui n'est pas complètement symétrique explique sans doute que les possesseurs de Rövö de la société Ntoro forment une élite peu nombreuse alors que les détenteurs de cloches de Mankombwu se sont largement multipliés. Cela prouve aussi que la règle habituelle de succession -- du père au fils aîné -- a été transgressée et que de nouvelles cloches ont été forgées probablement au profit de gendres ou de neveux utérins. Le fils d'un forgeron hérite normalement de la charge de son père, mais en cas d'absence d'héritier direct, le forgeron peut transmettre son savoir-faire et sa forge à son neveau utérin. Tout semble montrer qu'à une stratégie d'alliances matrimoniales s'est superposée une stratégie d'échanges entre sociétés initiatiques concourant à l'intégration de lignages immigrants parmi les lignages autochtones.

Sans entrer dans les détails, il faut relever que la société Wankya, dont les Rövö sont principalement des figurines de terre cuite, s'est également adjoint des calebasses Rö qui appartiennent aux deux sociétés primordiales. Mais contrairement à Ntoro, Wankya $n^{\prime}$ a rien cédé en échange aux autres sociétés Rö. Cette dernière société ne participe ni aux rites de fertilité, ni aux rituels de chasse, ni aux funérailles. Elle a reçu des autres mais ne leur a rien donné. Cette asymétrie est associée à un statut privilégié. La société wuli est acéphale. Or les initiés de Wankya y assument un rôle juridicopolitique embryonnaire. Il existe dans le village une aire sacrée unique où, en présence des Rövö de la société Wankya, leurs détenteurs peuvent "enterrer la pointe de lance", e'est-à-dire modifier la forme d'un rituel, l'interdire ou, au contraire, en créer un nouveau. Ce rituel d'"enterrement de la pointe de lance" est extrêmement rare, le dernier eut lieu en 1969. Cette année-là, on décela que la sorcelierie avait décimé beaucoup de gens et les initiés de Wankya, en accord avec le reste du village, décidèrent de transformer le rituel traditionnel de mise à mort d'un sorcier pour le rendre plus efficace. 
D'évidence, la sphère du Rö touche à tous les domaines de la vie religieuse, sociale et politique des Wuli. Au centre de cette gigantesque toile d'araignée veillent des objets aux formes étranges. Hétéroclites et venant sans doute d'horizons géographiques et symboliques divers, ils ont gardé les caractéristiques d'un passé déjà oublié ou transformé par le mythe, mais forment néanmoins sur un autre plan un ensemble unifié par le moule de la pensée symbolique wuli, dominée par la lutte contre la sorcellerie. Les hommes les façonnent pour qu'ils deviennent les réceptacles de forces surnaturelles contraignant ainsi ces dernières à se mettre au service de la société.

Pour désigner le statut des objets Rö, nous ne disposons dans la langue française que du terme malheureux de "fétiche". Nous avons hésité à l'employer du fait de ses connotations péjoratives. Il conviendrait fort bien cependant à condition de le purifier de son passé colonial et de lui restituer son sens étymologique strict d'objet rituel façonné par l'homme à des fins magico-religieuses, bénéfiques ou maléfiques.

Viviane Baeke

Université Libre de Bruxelles

\section{Références}

Baeke V.

1984 "Sorcellerie, sociétés secrètes et sacrifice chez les Wuli", Systèmes de pensée en Afrique noire, 7, 155-174

Dilion R.G.

1973 Ideology, process and change in pre-colonial Meta political organization (United Republic of Gameroon), University of Pennsylvania, Ph.D (University Microfilms, Ann Arbor, Michigan).

Jespers Ph.

1976 "Contribution à l'étude des autels sacrificiels du Nya chez les Minyanka du Mali", Systèmes de pensée en Afrique noire, 2, 111-139

Gebauer $P$.

1979 Art of Gameroon, Portland Art Association 


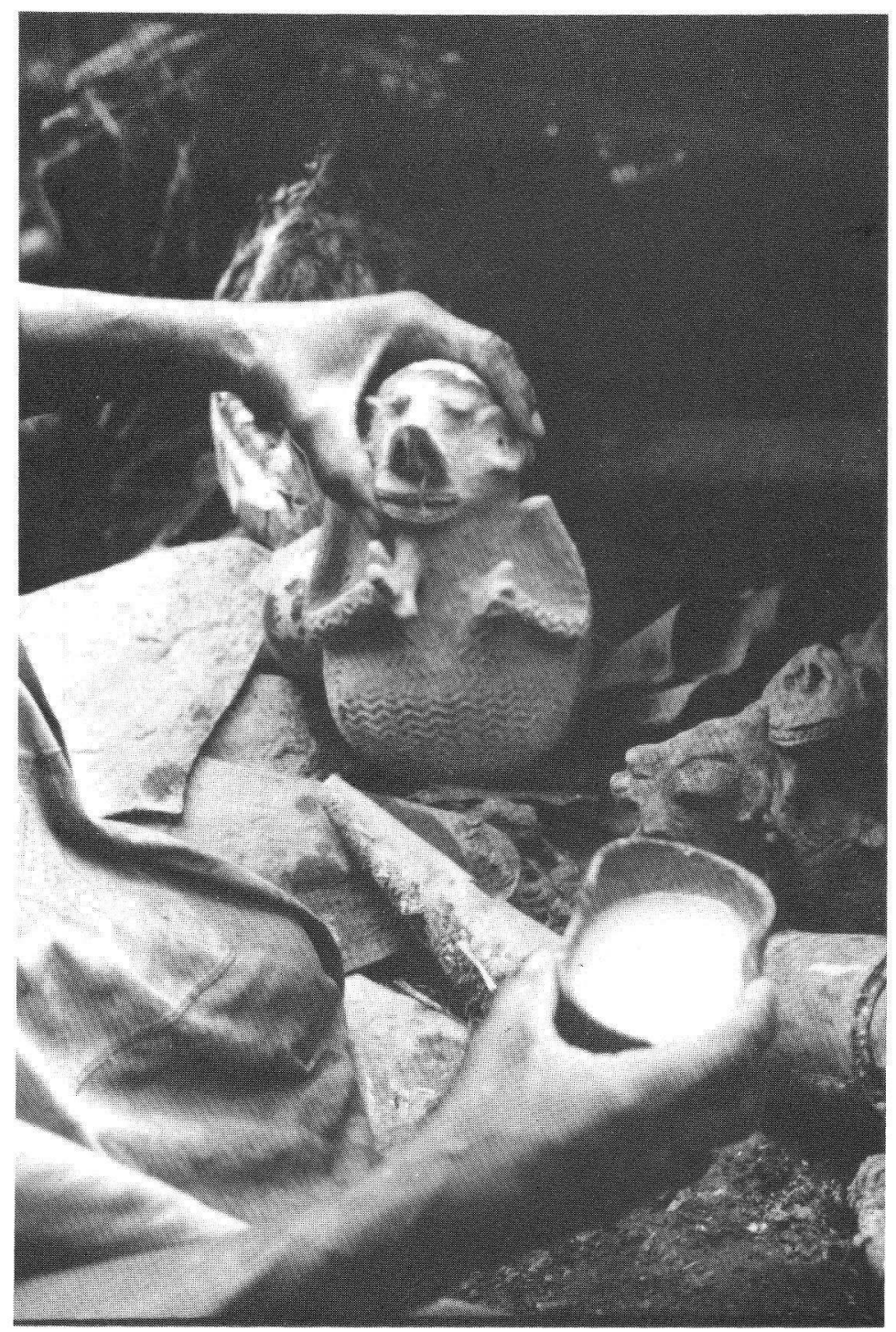

Gwofuru : une des sept figurines de la société wankya (terre cuite) 


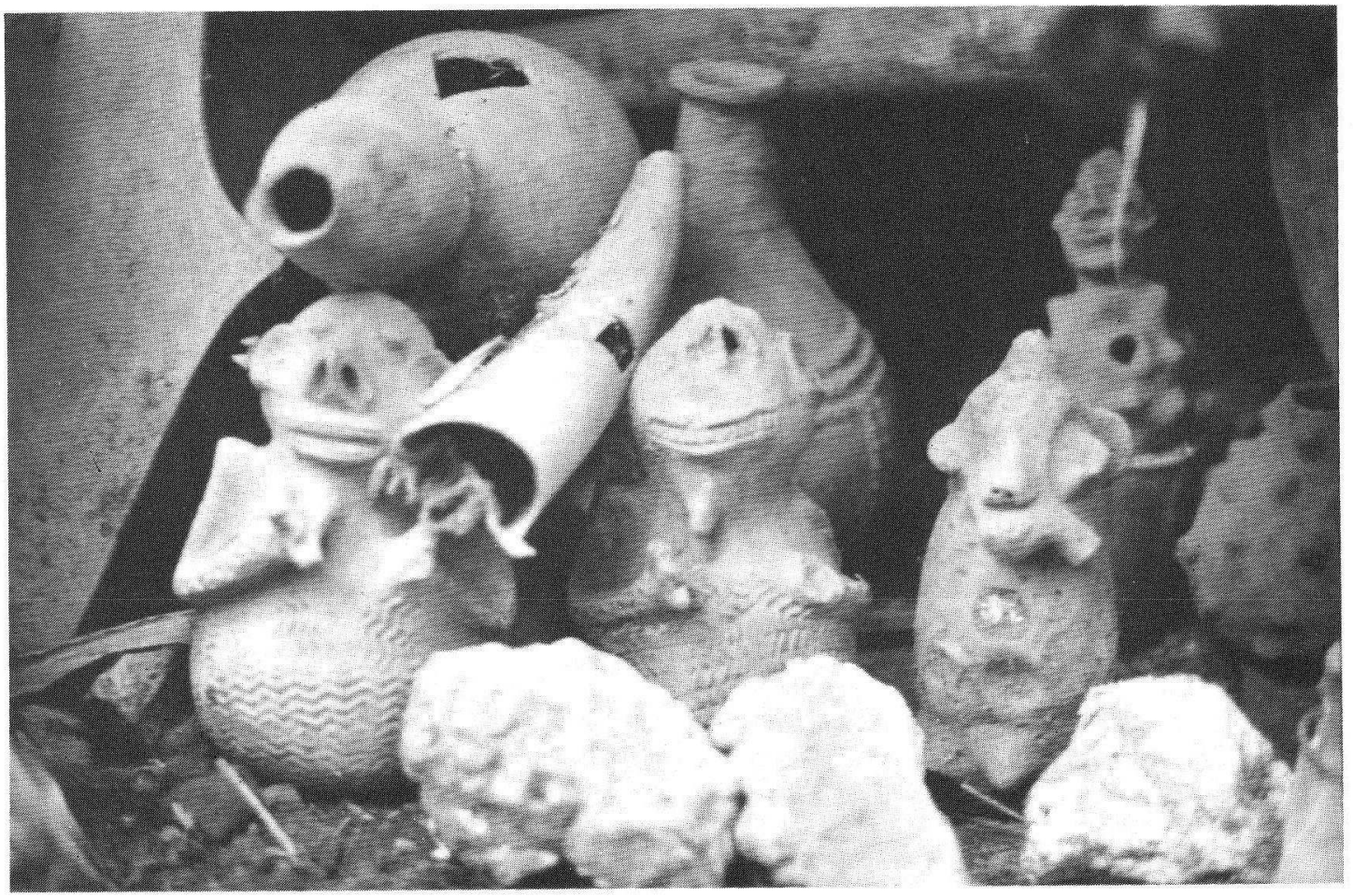

Figurines du sanctuaire de Nodza 